\title{
VIRTUAL PAINTING/WRITING WITH HAND GESTURE USING SIXTH SENSE DEVICE
}

\author{
Akash Sunil Gaikwad $^{1}$, V.Ajeeth Suryash ${ }^{2}$, Ragini M Daithankar ${ }^{3}$ \\ ${ }^{I}$ B.Tech, Electronics \& Communication Engineering, NIT Agartala, Tripura, India \\ ${ }^{2}$ BS-MS, Physics, NIT Agartala, Tripura, India \\ ${ }^{3}$ B.E, Computer Engineering, DRIEMS, Maharashtra, India
}

\begin{abstract}
Generally, we use pen and paper to scribble. But now a days we are writing / painting in digital form, that is we use laptops or our desktops. We are confined in the pen paper or digital computer screens to write or read anything, which is in digital form. We cannot directly interact with this digital data. In this paper we have discussed how we can directly interact with digital data using human hand gesture. This modern technology with which interaction of human with computer is made realistic rather than the old formal way (typing over keyboard or pointing through mouse) is called sixth sense technology.
\end{abstract}

Keywords: Sixth Sense Technology, Color detection, Image processing, Gestural Interaction, Augmented Reality, Wearable Interface etc...

\section{INTRODUCTION}

\section{What is sixth sense?}

Each one of us is aware of the five basic senses - Sight, Hearing, Taste, Smell and Touch. We are using this senses to sense the physical world and by analyzing this senses we properly interact with the physical world. These senses are used for modifying our interaction with the real world. As in Modern era there are information in digital form like sources like internet laptop, PDA, Smartphone, our natural senses can't analyze digital information from these sources. To interact with these kinds of information, we require a new type of sense which can efficiently map digital information. So we can develop a new type of sense known as "Sixth sense" that will work as an additional sense to our five natural senses. Although we have a different version of a computer that can interact with the digital data/ information, but there is not a proper system which can directly form link between digital information and our physical interaction. And to form a bridge for we developed sixth sense device, a system that can directly interact with the physical world and digital world. One of the important things of our body is our physical gesture which is most frequently used for interaction. We can use the same via particular system for Interaction with Digital Environment. There is a lot of complexity and flexibility of this physical interaction, and it is very hot topic in the research field. To recognize these physical gestures efficiently, Sixth sense device is used. In this way of interaction, the command to the computer is given through hand gesture.

This project combines the joint venture of the following components camera for capturing video or sequence of frames, physical hand gesture cladded with color marker, computing device for processing and analyzing the data, projector for projecting visual information to any physical surface, and mirror for reflecting purpose. This whole system is called sixth sense wearable interface device (which a pendant like wearable device). And using this device we can virtually write or paint on any tangible surface without using any physical equipment like pen paper. In this paper, we mainly focus at the application of sixth sense technology to virtually write or paint which results in an augmented reality.

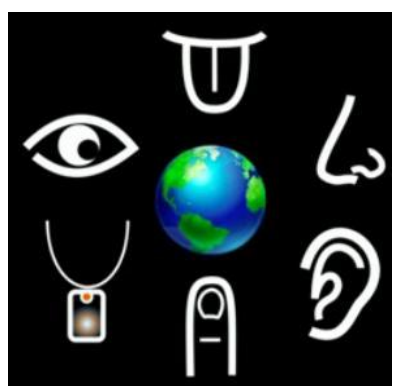

Fig-1: Sixth Sense

\section{COMPONENTS OF DEVICE/SYSTEM:}

\subsection{Camera}

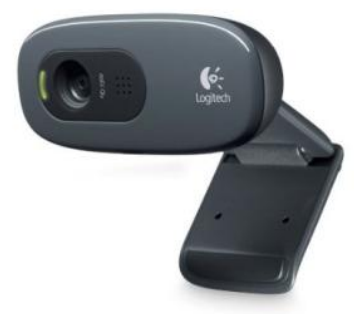

Fig-2: Camera 
It captures the image continuously at high frame rates to form a video. It is also considered as the eye of the system, which helps the system to interact with the real world information. It is placed in pendent like device in such a way that, it can easily sense our gestures. It also sends the captured frames to the computing device instantaneously.

\subsection{Projector}

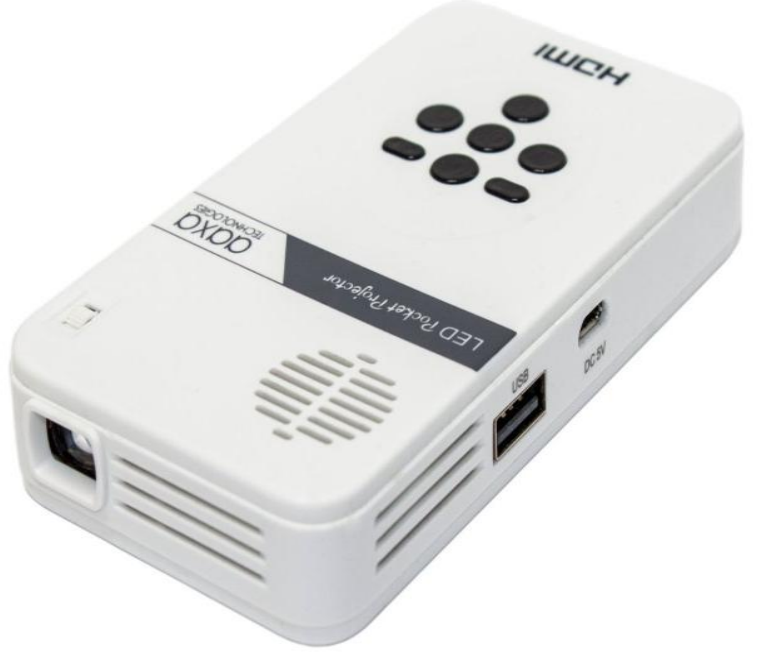

Fig -3: Projector

It is a mini LED Pico-projector, powered by a battery. It is connected to the computing device by an HDMI cable. It projects the visual information sent from the computing device on any available surface. It displays the digital information (visual information) to real world. It is placed in the plane of pendent.

\subsection{Mirror}

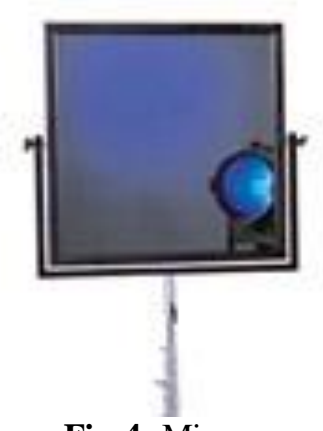

Fig-4: Mirror

It plays a vital role in projection of visual information. As the projector is facing downwards, the mirror is used to direct the rays coming out of projector to any appropriate surface. To facilitate its function, it is placed at an angle of $45^{0}$ to the plane of projector.

\subsection{Computing Device}

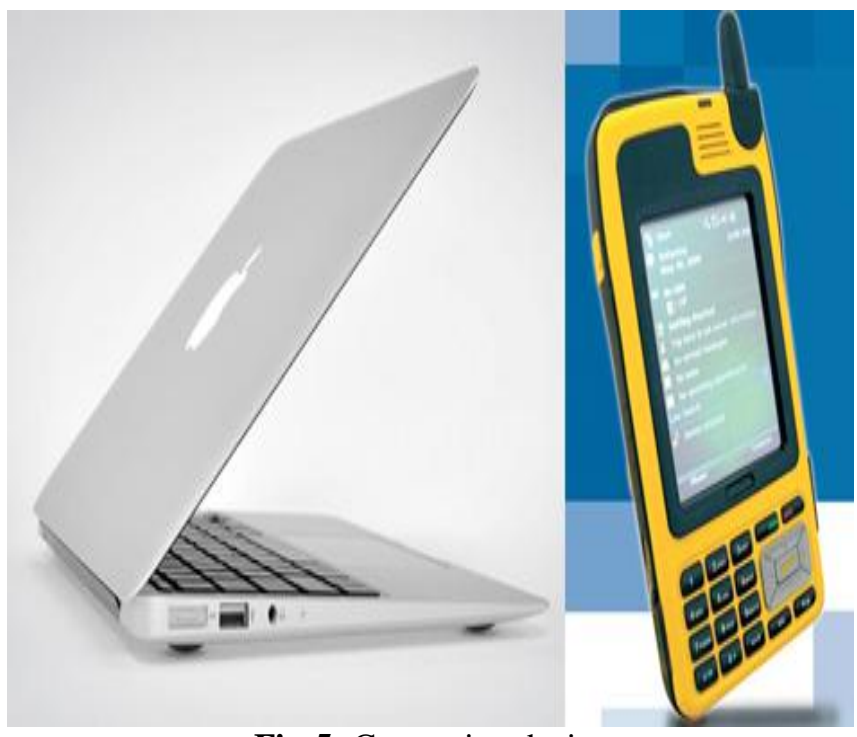

Fig-5: Computing device

It is the heart of the system. It can be any portable device like smartphones (like Apple), laptops or Raspberry-pi computers. It is placed in user's pocket. It makes use of specially designed software in which our algorithm is processed.

\subsection{Coloured Pointer Caps}

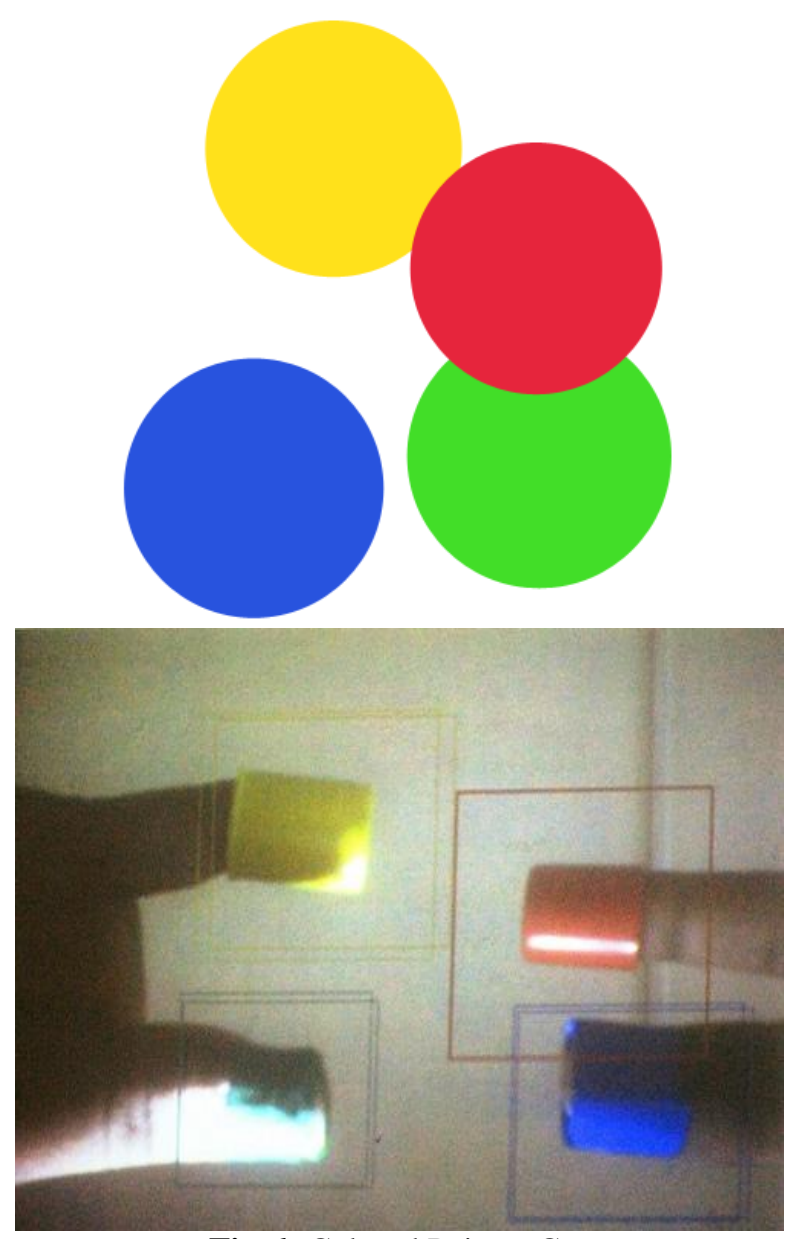

Fig-6: Colored Pointer Cap 
They are placed over the tip of the user's fingers. The color of the markers can be any bright distinct color such as Red, Green and Blue. The movements and the arrangements of the markers are interpreted into a gesture. These are used for giving commands to the computing device.

\section{CONSTRUCTION AND WORKING}

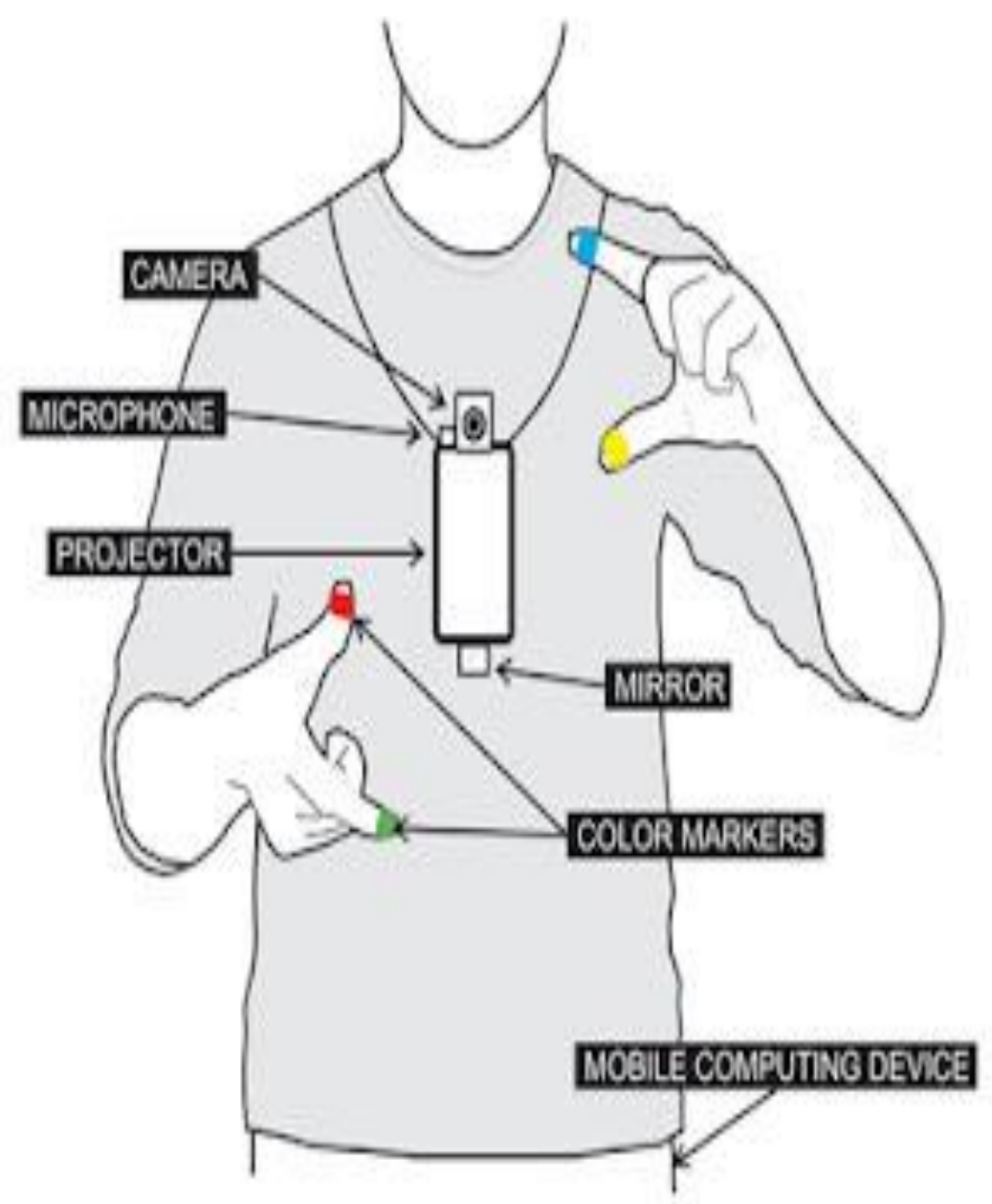

Fig-7: Prototype

The total system works on the sixth sense device. System contains several components like mini Led Projector, portable computing device, colored cap, camera \& mirror. Except the computing device, the other components are placed in a pendent like device for a convenient interaction. Camera is placed horizontally to cover the maximum field of view. Projector is placed such a way that it faces downwards in the plane of pendent. Mirror is placed at $45^{\circ}$ from the plane of pendent to horizontally direct the optical rays of projector. The computing device is placed in our pocket. The colored pointer caps are placed over the fingertips. 


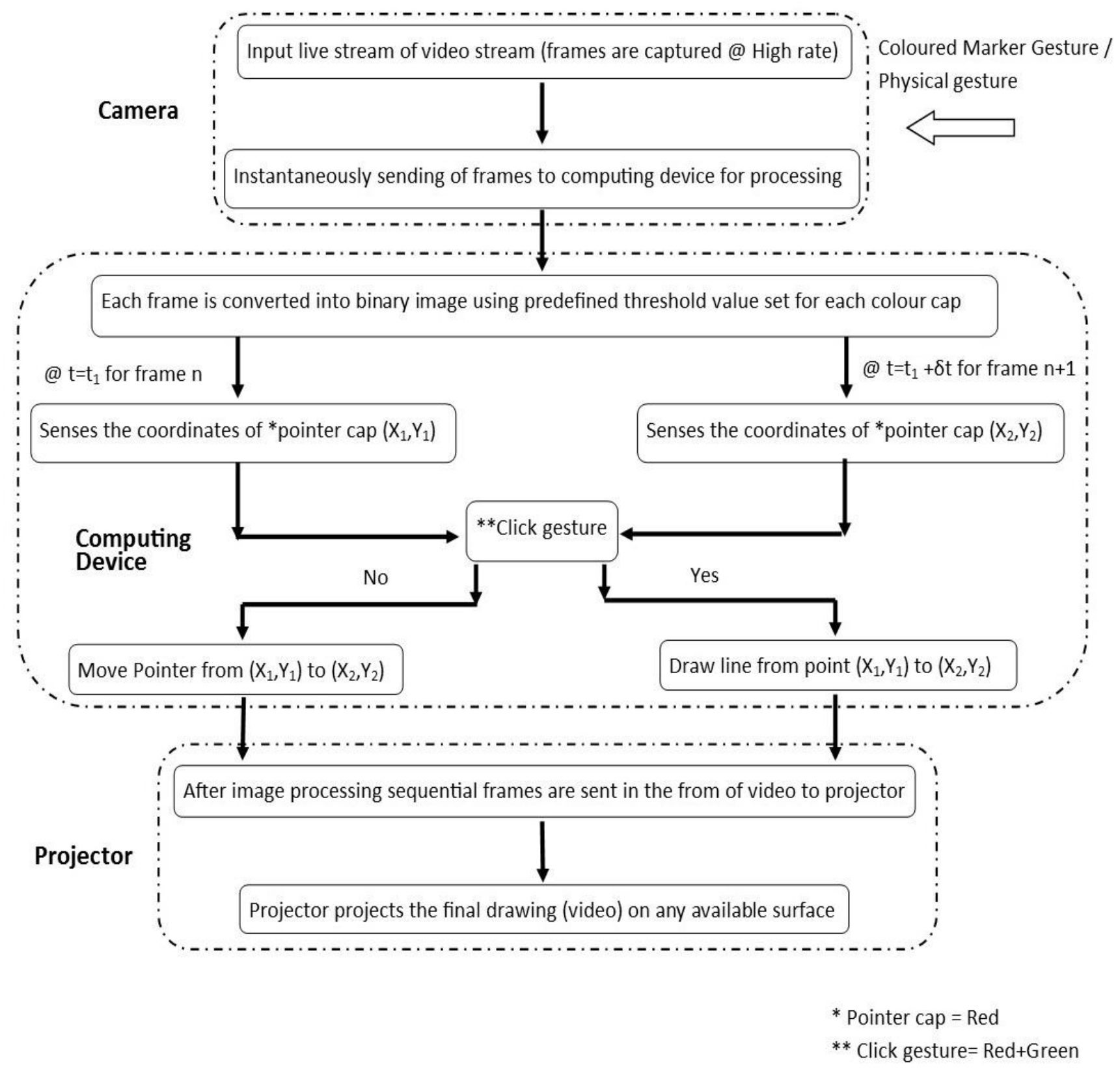

Fig. Block Diagram of Virtual Painting/Writing with hand gestures using Sixth Sense Device

The camera captures frames at high rates and converts them into input live stream. It takes real time video coverage of the hand movements/gestures and sends them instantaneously to computing device for processing. Now these frames are processed by applying suitable image processing techniques. Each frame is converted into a binary image with the use of predefined threshold value set for each colored cap. Now the coordinate sensing algorithm is applied. Consider at time $t_{1}$, the frame numbered $\mathrm{N}=\mathrm{n}_{1}$ is processed and the computational device senses the coordinates of colored pointer cap in $2 \mathrm{D}$-plane. Let it's coordinates be $\left(\mathrm{X}_{1}, \mathrm{Y}_{1}\right)$. For time $\mathrm{t}_{1}+\delta \mathrm{t}$ the frame numbered $\mathrm{N}=\mathrm{n}_{1}+1$ is processed and the computational device senses the coordinates of colored pointer cap. Let the coordinates be $\left(\mathrm{X}_{2}, \mathrm{Y}_{2}\right)$. If the click gesture is recognized, the processor draws line from point $\left(\mathrm{X}_{1}, \mathrm{Y}_{1}\right)$ to $\left(\mathrm{X}_{2}, \mathrm{Y}_{2}\right)$ on the screen. [The click gesture is the state when processor senses two colored caps in a single frame. For example, red and green]. Else the processor moves the cursor from point $\left(\mathrm{X}_{1}, \mathrm{Y}_{1}\right)$ to $\left(\mathrm{X}_{2}, \mathrm{Y}_{2}\right)$. This process goes on continuously. After this process we get final sequence of frames and these frames are sent to projector and projected in the form of video on any available surface. 
File Edit Tert Go Cell Tools Debug Desktop Window Help

$x \times x$

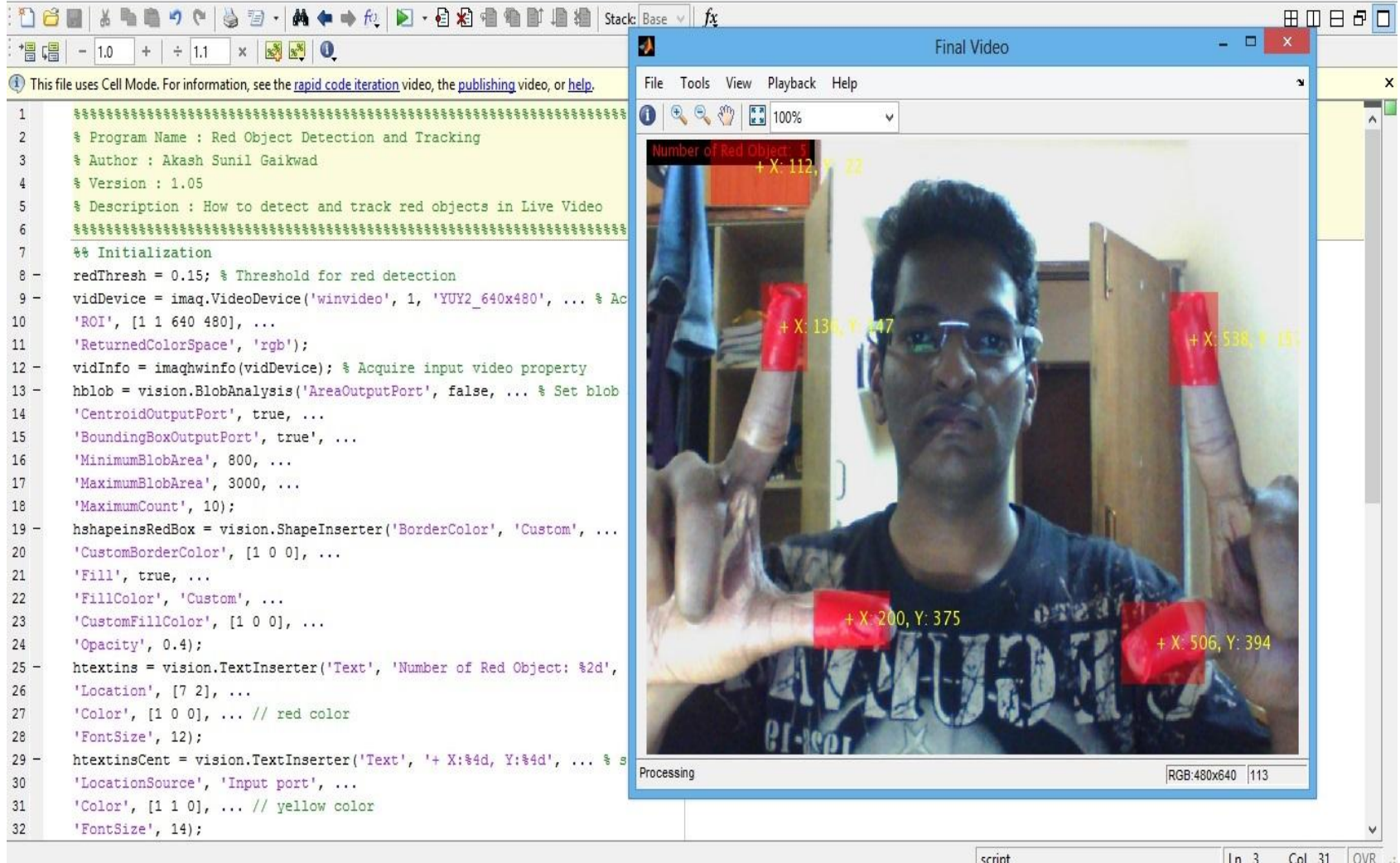

Fig-8: Color Marker Detection

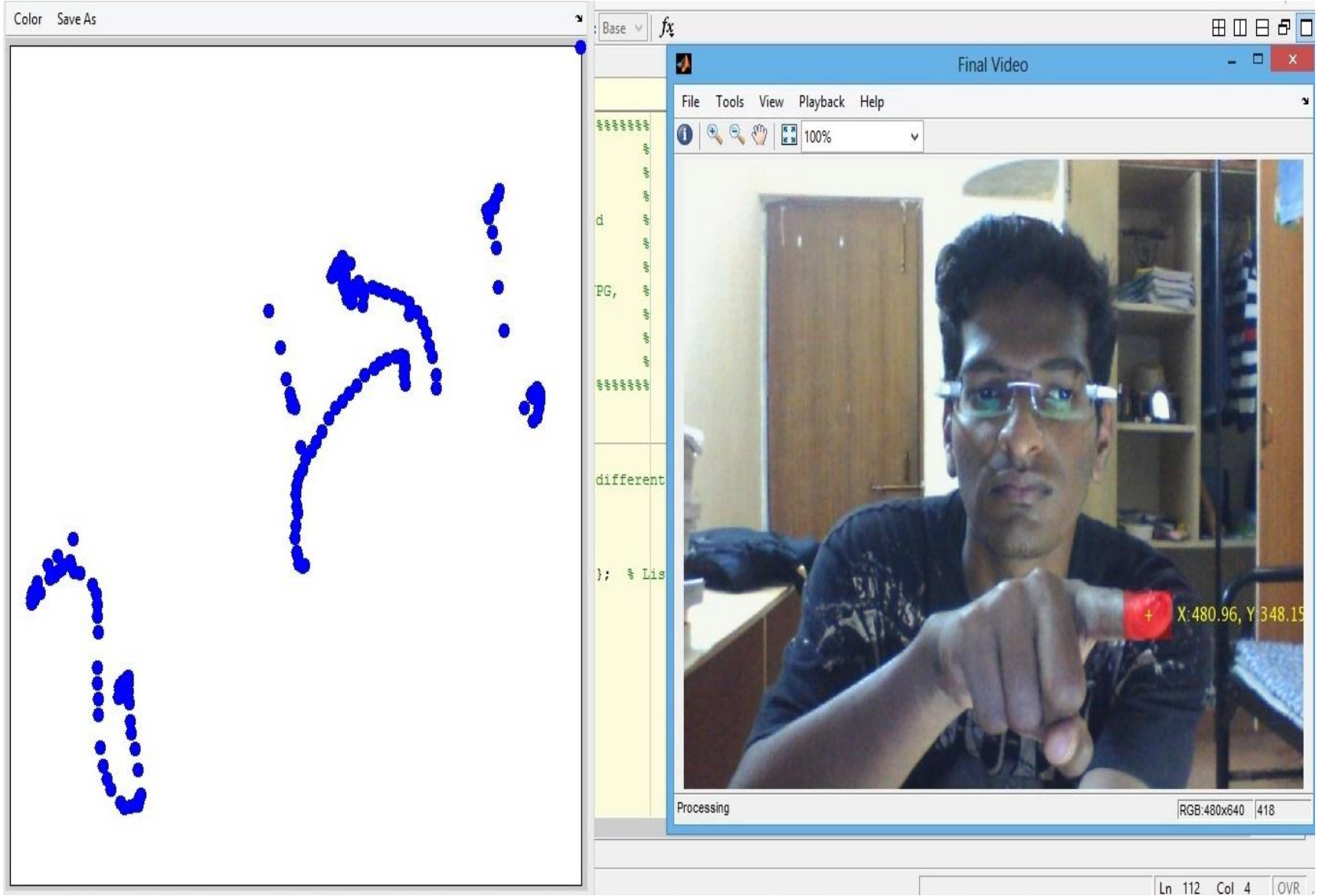

Fig-9: Virtual Painting/Writing 


\section{FEATURES AND APPLICATION}

- Virtual painting using sixth sense technology supports free hand drawing.

- $\quad$ This method of virtual writing can be implemented in virtual class rooms and conferences.

- It can replace our traditional pen-papers and boardmarkers with only color cladded caps, which are easy and efficient to use.

- The whole system is portable, such that it can be worn like a pendent around our neck.

\section{CONCLUSION}

This technology helps us to get rid of formal way of interaction to our digital data. It proves to be the faster way of recording and accessing our data, compared to current scenario. The data storage, as soft copy makes it easily transferable and occupies much lesser space than the traditional way. The content duplication is much simpler. All this advantages are incorporated with hand gestures as input commands, which makes the whole system to be more realistic. It gives us hope towards global digitalization.

\section{REFERENCES}

[1]. Mistry, Pranav, Pattie Maes, and Liyan Chang. "WUW wear Ur world." Proceedings of the 27th international conference extended abstracts on Human factors in computing systems - CHI EA '09. Boston, MA, USA, 2009. 4111.

[2]. Meenakshi Gupta, Shruti Sharma, Virtual Class room using six sense Technology, IOSR Journal of Computer Engineering (IOSRJCE) ISSN: 2278-0661, ISBN: 22788727 Volume 6, Issue 4 (Sep. -Oct. 2012), PP 20-25

[3].http://www.ted.com/talks/pranav_mistry_the_thrilling_p otential_of_sixthsense_technology.html

Last visited on 10th Dec'14

[4].http://www.pranavmistry.com/projects/sixthsense/

[5]. Susmit Sarkar, Arindam Bose, Face Detection and Tracking System, International Journal of Scientific \& Engineering Research, Volume 3, Issue 10, October-2012 ISSN 2229-5518

[6].http://theviewspaper.net/sixth-sense-technology-willrevolutionize-the-world/

\section{BIOGRAPHIES}

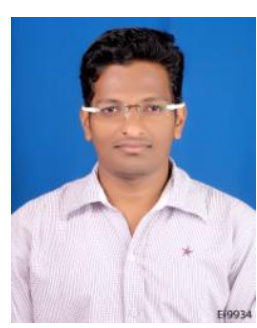

Akash Sunil Gaikwad currently in final year Pursuing (B.Tech) Bachelor of Technology (Electronics \& Communication Engineering) from "National Institute of Technology, Agartala.

Email Id -akashragi@gmail.com

Phone No-+919862102486

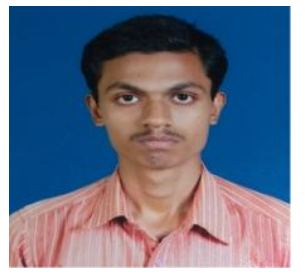

V.Ajeeth Suryash currently in second year Pursuing (BS-MS) Bachelor \& Master of Science (Physics Department) from "National Institute of Technology, Agartala. Email -ajeetheinstein@gmail.com Phone:+918413028010

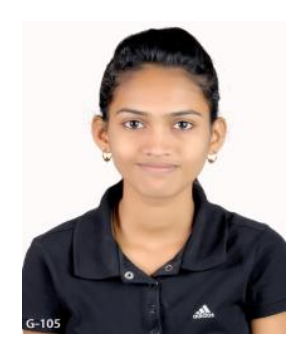

Ragini M Daithankar currently in third year Pursuing (B.E) Bachelor of Engineering (Computer Engineering) from "Dilkap Research Institute of Engineering and management studies, Neral.

Email:raginidaithankar01@gmail.com Phone No-+919730517429 